\title{
Bladder catheterization increases susceptibility to infection that can be prevented by prophylactic antibiotic treatment
}

\author{
Matthieu Rousseau, ${ }^{1}$ H.M. Sharon Goh, ${ }^{2}$ Sarah Holec, ${ }^{2}$ Matthew L. Albert, ${ }^{1}$ Rohan B.H. Williams, ${ }^{3}$ \\ Molly A. Ingersoll, ${ }^{1}$ and Kimberly A. Kline ${ }^{2}$ \\ 'Unité d'Immunobiologie des Cellules Dendritiques, Department of Immunology, Institut Pasteur and INSERM U1223, \\ Paris, France. ${ }^{2}$ Singapore Centre on Environmental Life Sciences Engineering, School of Biological Sciences, Nanyang \\ Technological University, Singapore. ${ }^{3}$ Singapore Centre for Environmental Life Sciences Engineering, National University \\ of Singapore, Singapore.
}

Catheter-associated urinary tract infections (CAUTI) are the most common hospital-associated infections. Here, we report that bladder catheterization initiated a persistent sterile inflammatory reaction within minutes of catheter implantation. Catheterization resulted in increased expression of genes associated with defense responses and cellular migration, with ensuing rapid and sustained innate immune cell infiltration into the bladder. Catheterization also resulted in hypersensitivity to Enterococcus faecalis and uropathogenic Escherichia coli (UPEC) infection, in which colonization was achieved using an inoculum 100 -fold lower than the $I_{90}$ for infection of an undamaged urothelium with the same uropathogens. As the time of catheterization increased, however, colonization by the Gram-positive uropathogen $E$. faecalis was reduced, whereas catheterization created a sustained window of vulnerability to infection for Gram-negative UPEC over time. As CAUTI contributes to poorer patient outcomes and increased health care expenditures, we tested whether a single prophylactic antibiotic treatment, concurrent with catheterization, would prevent infection. We observed that antibiotic treatment protected against UPEC and $E$. faecalis bladder and catheter colonization as late as 6 hours after implantation. Thus, our study has revealed a simple, safe, and immediately employable intervention, with the potential to decrease one of the most costly hospital-incurred infections, thereby improving patient and health care economic outcome.

Authorship note: M. Rousseau and H.M.S. Coh contributed equally to this work. M.A. Ingersoll and K.A. Kline contributed equally to this work.

Conflict of interest: The authors have declared that no conflict of interest exists.

Submitted: April 25, 2016 Accepted: August 23, 2016 Published: September 22, 2016

Reference information: JCI Insight. 2016;1(15):e88178. doi:10.1172/ji.insight.88178.

\section{Introduction}

Urinary tract infections (UTI) are the most common hospital-associated infection; $75 \%$ of these cases are associated with urinary catheterization $(1,2)$. Approximately $15 \%-25 \%$ of all hospitalized individuals and $5 \%-10 \%$ of those living in long-term care facilities receive a urinary catheter during their stay (3). Of those, $26 \%$ experience bacteriuria and $6 \%$ will have a symptomatic catheter-associated UTI (CAUTI) within 10 days of catheterization (4). Individuals with CAUTI typically exhibit longer recovery times and hospital stays and increased morbidity and mortality (5-8). In the US, there are more than one million CAUTI each year, associated with nearly $\$ 600$ million in annual health care costs $(9,10)$.

Urinary catheterization, in the absence of bacterial infection, is associated with sterile inflammation, histological abnormalities, and edema $(11,12)$. In a mouse model of bladder catheterization, edema and plasma protein extravasation occur within 3 hours of catheter implantation into the bladder. Subsequently, epithelial cell shedding, cytokine production, and myeloid cell infiltration are observed by 24 hours post- catheterization (hpc) $(13,14)$. The pathways leading to catheter-induced sterile inflammation are not well understood. Dexamethasone-mediated immunosuppression abrogates acute catheter-induced inflammation in a mouse model for up to 9 hours but does not affect long-term catheter-associated edema and tissue damage (13). Moreover, inhibitors of the neurogenic inflammatory pathway have no effect on catheter-associated inflammation, indicating that chronic inflammation is independent of both glucocorticoid and neurogenic inflammatory pathways (13). 
A

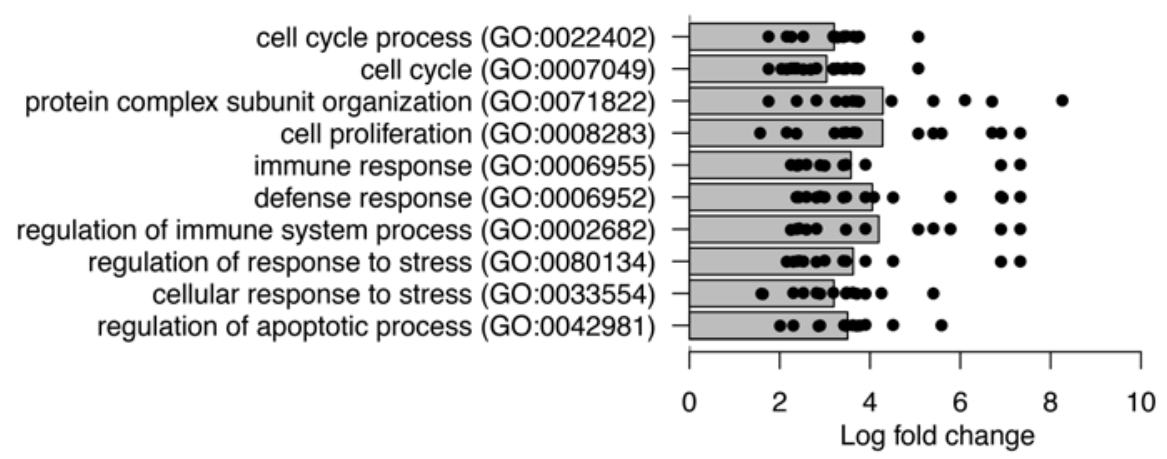

B

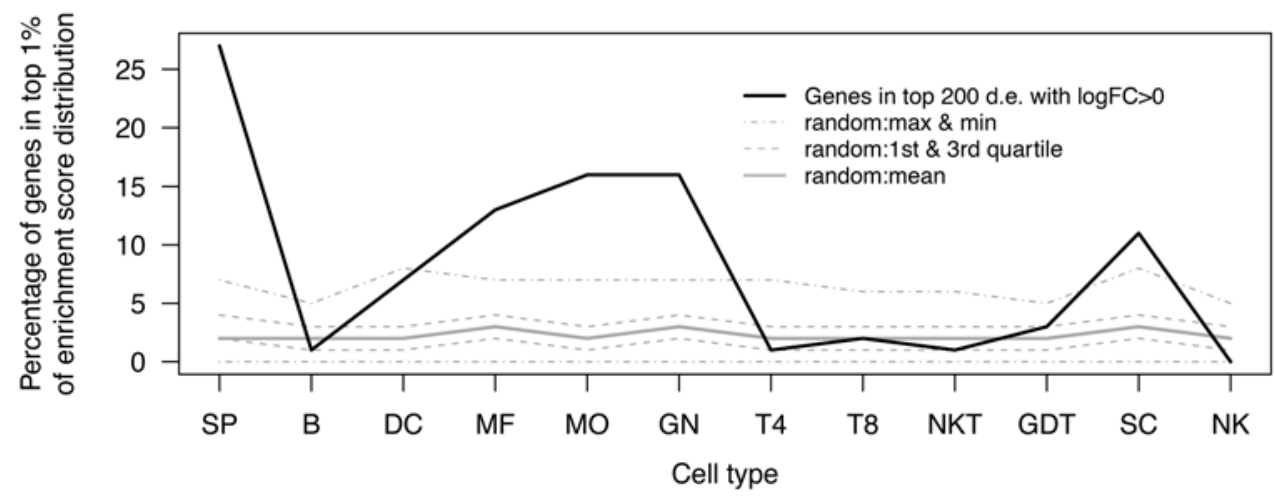

Figure 1. Catheterization induces substantial gene expression changes in the bladder. Female C57BL/ 6 mice were implanted with a 5-mm silicon catheter, which naive control animals did not receive. After 24 hours, bladders were processed and RNA was recovered. Gene expression analysis was performed as described in the Methods. (A) Summary of gene ontology (GO) enrichment analysis in the top 200 most differentially expressed genes $\left(P^{\mathrm{adj}}<5 \times 10^{-26}\right)$ that showed increased mRNA levels in catheterized versus noncatheterized bladders. Each data point shows the $\log _{2}$ mean expression ratio between catheterized and noncatheterized animals ( $x$ axis). Genes are categorized by annotation to each enriched GO biological process term (y axis). Gray bars show the mean log fold change for genes annotated to each enriched term. See Supplemental Table 4 for further results. (B) To identify differentially expressed genes (d.e.) associated with specificity for a given cell type in the ImmGen data, we calculated the percentage of the top 200 genes that was contained in the top $1 \%$ of the distribution of the cell-type-specific enrichment score (51) (solid black line; compared with 100 sets of size- and abundance-matched random genes [summarized by gray lines]). SP, stem and progenitor cells; B, B cells; MF, macrophages; MO, monocytes; GN, granulocytes; T4, CD4+ cells; T8, CD8+ cells; GDT, $\gamma \delta$ T cells; SC, stromal cells. See Supplemental Tables 1-5 and Supplemental Figures 1-3 for related analyses. The experiment was performed twice ( $n=3$ mice per group/experiment). Representative data are shown from one experiment.

Uropathogenic Escherichia coli (UPEC) is associated with $>80 \%$ of all uncomplicated UTI, i.e., in the absence of urinary catheter. However, in the presence of a urinary catheter, the spectrum of infecting bacterial species shifts such that UPEC accounts for approximately $50 \%$ of CAUTI, and uropathogens less commonly associated with uncomplicated UTI become more prevalent (15). Indeed, despite the robust inflammatory response associated with urinary catheterization, many bacterial species colonize the urinary catheter and persist within biofilms, which are intrinsically tolerant to host clearance. For example, Enterococcus faecalis is associated with approximately $5 \%$ of uncomplicated UTI but $15 \%-30 \%$ of CAUTI (16). In a mouse model, the strong proinflammatory response elicited during CAUTI is not sufficient to limit infection when initiated at the same time as catheterization $(13,14)$. Instead, fibrinogen, released into the bladder lumen during acute inflammation, coats the catheter surface and is directly bound by the Ebp pili of E. faecalis, promoting bacterial attachment and biofilm formation (17). Thus, E. faecalis UTI is significantly augmented by catheterization. Notably, treatment with dexamethasone or depletion of circulating neutrophils enhances acute $E$. faecalis colonization of bladders and catheters, illustrating that immune infiltration is important for bacterial clearance and suggesting that suppression of inflammation may result in increased susceptibility to CAUTI (13).

This observation prompted us to hypothesize that catheter-associated inflammation may create a window of susceptibility to infection in an otherwise healthy organ. To test this, we first characterized the 
A

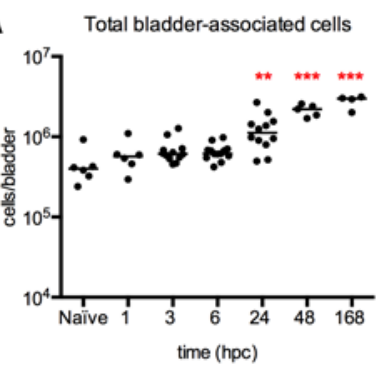

B Gated on:
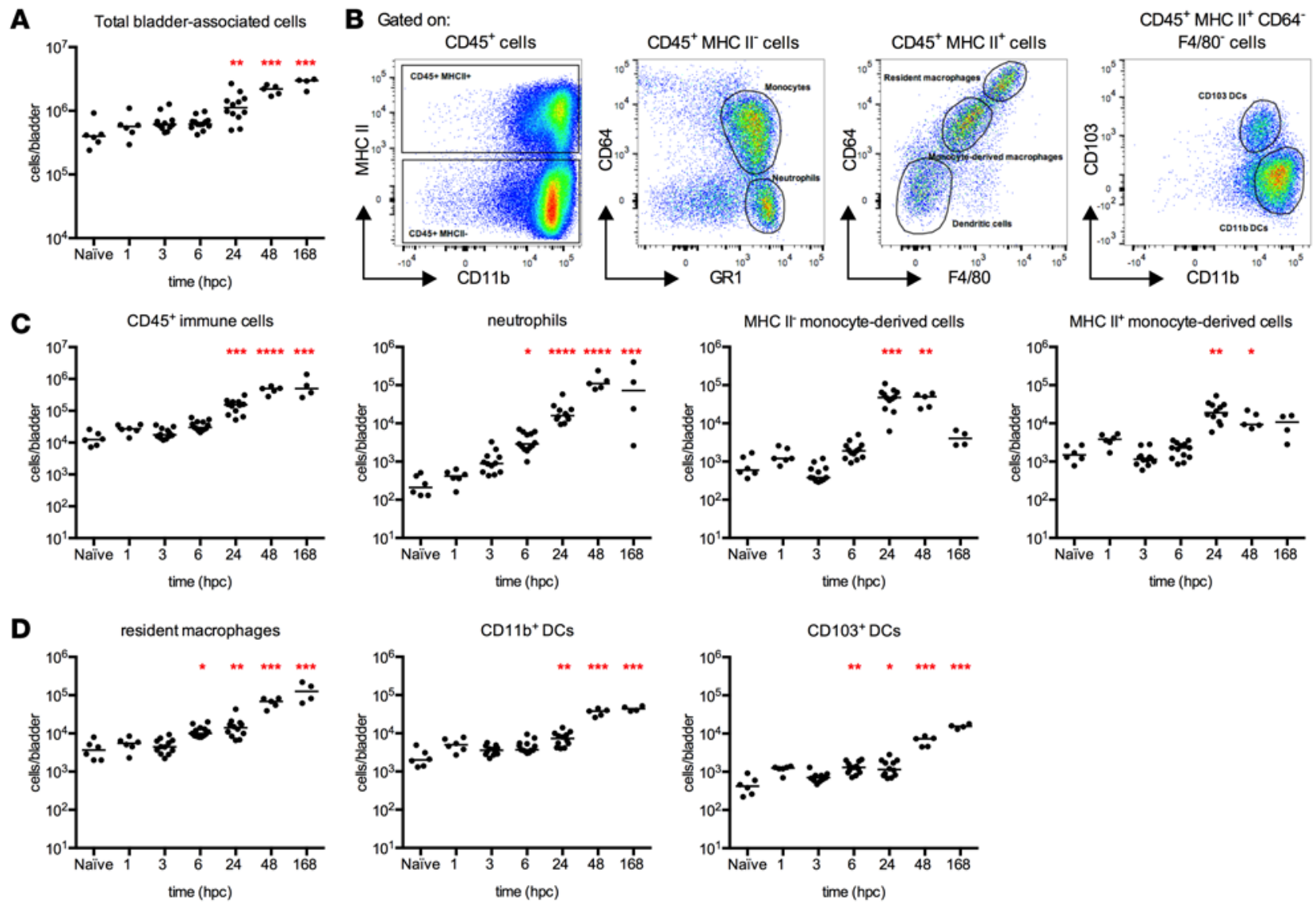

CD11b+DCs

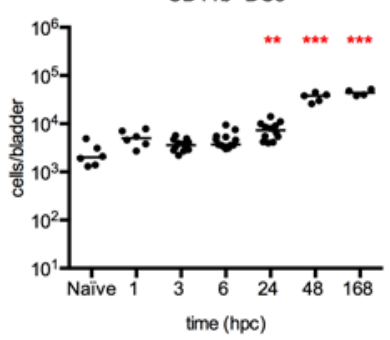

$\mathrm{CD}_{103^{+} \mathrm{DCs}}$

E
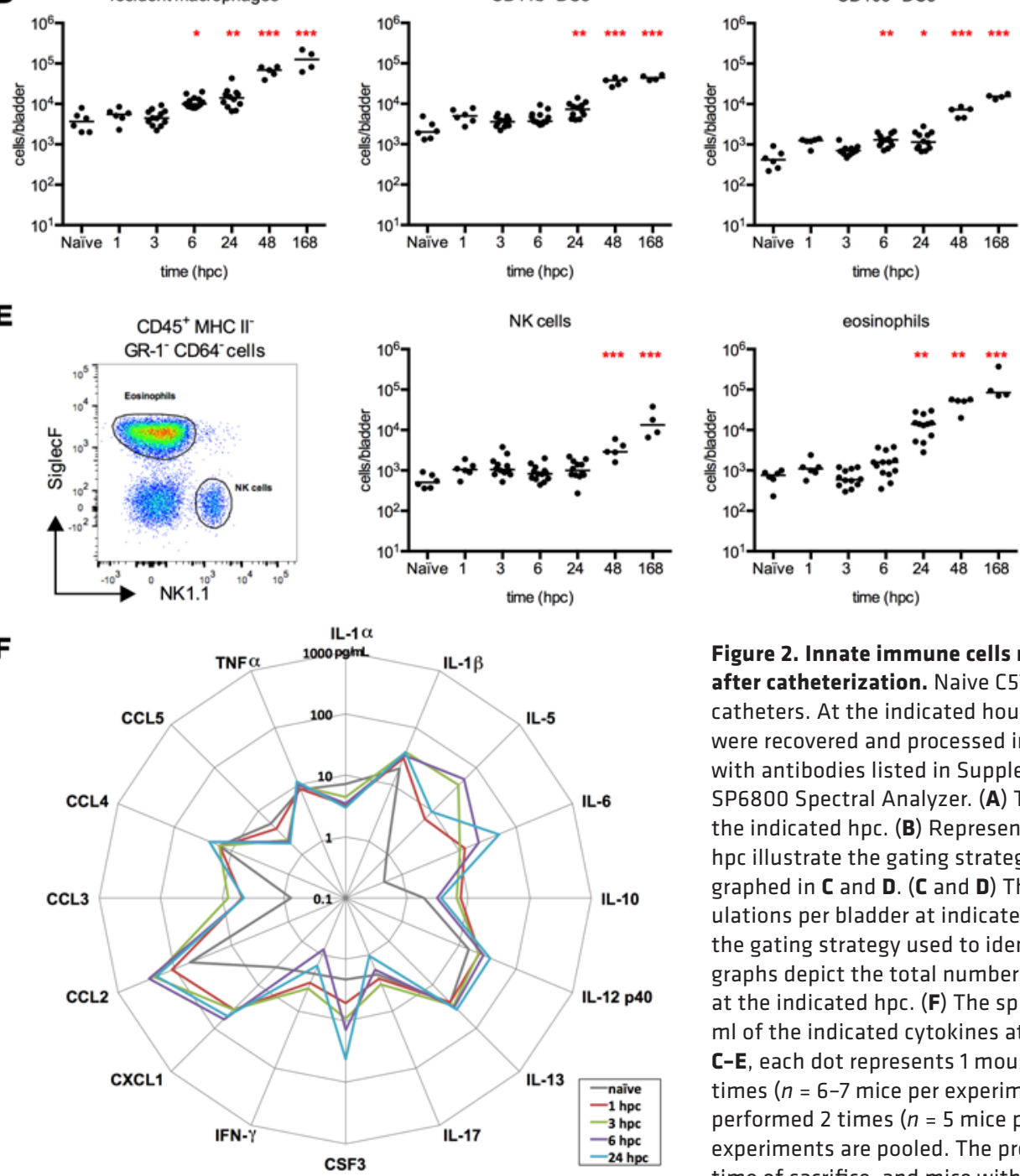

Figure 2. Innate immune cells robustly migrate into the bladder rapidly

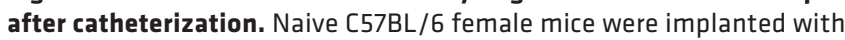
catheters. At the indicated hour after catheterization (hpc), bladders were recovered and processed into a single-cell suspension, labeled with antibodies listed in Supplemental Table 6, and acquired on a Sony SP6800 Spectral Analyzer. (A) The total number of cells per bladder at the indicated hpc. (B) Representative dot plots from a mouse 24 or 48 hpc illustrate the gating strategy used to identify immune cell subsets graphed in $\mathbf{C}$ and $\mathbf{D}$. (C and $\mathbf{D}$ ) The total number of the specified cell populations per bladder at indicated hpc. (E) The dot plot (48 hpc) illustrates the gating strategy used to identify NK cells and eosinophils, and the graphs depict the total number of NK cells and eosinophils per bladder at the indicated hpc. (F) The spider plot depicts absolute values in pg/ $\mathrm{ml}$ of the indicated cytokines at $\mathrm{O}$ (naive), 1, 3, 6, and $24 \mathrm{hpc}$. In $\mathbf{A}$ and C-E, each dot represents 1 mouse, and the experiment was performed 2 times ( $n=6-7$ mice per experimental group). In $\mathbf{F}$, the experiment was performed 2 times ( $n=5$ mice per experimental group). In $\mathbf{A}$ and $\mathbf{C}-\mathbf{F}$, experiments are pooled. The presence of a catheter was verified at the time of sacrifice, and mice without a catheter were excluded from the analysis. ${ }^{*} P<0.05,{ }^{* *} P<0.01,{ }^{* * *} P<0.001,{ }^{* * * *} P<0.0001$ for experimental groups compared with the control naive group, Kruskal-Wallis test with Dunn's post-test to correct for multiple comparisons. 
response to catheter implantation over time to define the immune signature and potential for bacterial colonization. We observed that, despite a robust innate immune cell infiltration, both E. faecalis and UPEC colonized catheterized bladders more efficiently than uncatheterized bladders. Furthermore, UPEC colonization was significantly increased when infection was initiated 6 hpc. By contrast, E. faecalis colonization diminished when infection was initiated after catheter implantation rather than concurrently. A simple treatment, consisting of a single antibiotic injection was able to completely inhibit bladder and catheter colonization, providing a potential clinical intervention that may positively affect patient outcomes and health care costs.

\section{Results}

Catheterization induces marked global gene expression changes in the bladder. To better understand the spectrum of host responses to urinary catheterization and how those responses may affect susceptibility to infection, we implanted urinary catheters into mice and performed RNA expression profiling on whole bladders after 24 hours (Supplemental Figure 1, A-D, and Supplemental Table 1; supplemental material available online with this article; doi:10.1172/jci.insight.88178DS1). Of the 14,226 detectable genes, 4,695 (33\%) demonstrated statistically significant changes in mRNA levels between the catheterized and naive state, with an adjusted $P$ value $\left(P_{\text {adj }}\right)$ of 0.05 . Of these, the top-ranked 100, 200, 500, 1,000, and 1,500 differentially expressed transcripts were observed at $P_{\text {adj }} \approx 10^{-36}, P_{\text {adj }} \approx 10^{-26}, P_{\text {adj }} \approx 10^{-16}, P_{\text {adj }} \approx 10^{-9}$, and $P_{\text {adj }} \approx 10^{-6}$, respectively. Notably, significant overlap in the top-ranked differentially expressed transcripts was observed between two separate biological experiments (Supplemental Table 2 and 3). We examined the biological processes associated with these differentially expressed genes using enrichment analysis of gene ontology (GO) biological process annotations and observed that GO terms related to cell proliferation, cell migration, apoptosis, movement of cellular components, and immune system-related processes were significantly enriched $\left(P_{a d j}<0.05\right.$; Fisher's exact test, corrected for number of terms tested) in the top 200 differentially expressed genes (Figure 1A; see also Supplemental Table 4 for extended analyses in expanded sets of topranked differentially expressed genes).

The strong immune-related signature in catheterized bladders, such as granulocyte infiltration, was consistent with features of sterile inflammation (18). Thus, we hypothesized that specific immune cell populations were present in the tissue and that genes associated with these populations would be prominently represented in the differentially expressed genes we observed to be upregulated in the catheterized state. We examined publicly available data from a compendium of immune cell types in C57BL/6J mice collected by the Immunological Genome Project (ImmGen) $(19,20)$ and observed that this was indeed the case. Of the 81 gene sets defined by ImmGen that are associated with specific classes of immune and nonimmune cell types (coarse modules, as defined in ref. 19), 17 displayed significantly higher expression in catheterized mice compared with naive control animals $\left(P_{\text {adj }}<0.05\right.$; corrected for number of modules tested, against the null hypothesis of no overall change in expression; Supplemental Figure 2 and Supplemental Table 5). These modules were associated with specific immune cell lineages, including DCs and granulocytes, consistent with the involvement of biological processes such as cell migration and motility. The additional presence of mixed use modules and downregulated with differentiation modules prompted us to consider that additional cell types may be induced to migrate and differentiate in response to catheterization. Indeed, in the top 200 and 500 gene sets, genes specific for macrophages, monocytes, and granulocytes were overrepresented and showed increased mRNA levels in catheterized versus naive animals, suggesting these cells infiltrate the bladder during catheterization (Figure 1B and Supplemental Figure 2). Notably, and consistent with changes observed during sterile inflammation (18), genes strongly upregulated in the catheterized state were also identified within stromal cell types (Figure 1B and Supplemental Figure 3).

Catheter implantation stimulates robust and sustained immune infiltration. To gain greater insight into the kinetics and composition of the predicted immune cell infiltration, we analyzed cellular migration into the bladder from 1 hour to 1 week after catheter implantation by spectral flow cytometry. Bladders from naive or catheterized mice were digested to a single-cell suspension and immunostained for predefined immune cell markers (21). We chose to use spectral analysis, which permitted investigation of a greater number of parameters than possible using conventional flow cytometry, including natural autofluorescent signals, without the need for compensation (22). We incorporated the strongest autofluorescent signal emitted from inflamed bladder tissue into the deconvolution algorithm to exclude autofluorescent nonimmune cells, whose signal typically spills over into the channels used to identify CD $45^{+}$immune cells (21). 

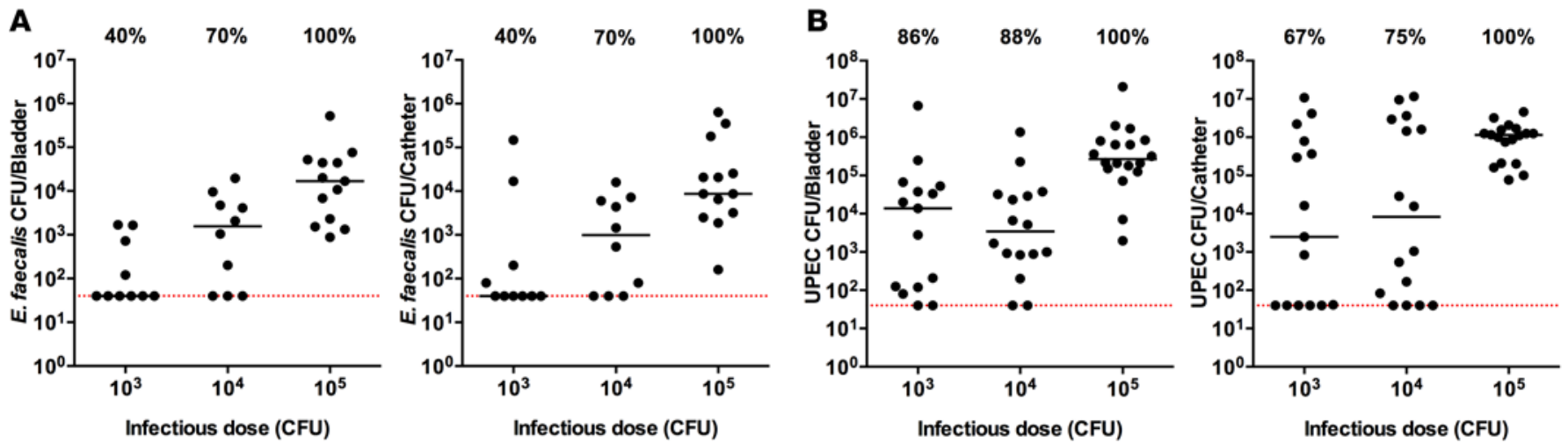

C Catheterization time before infection

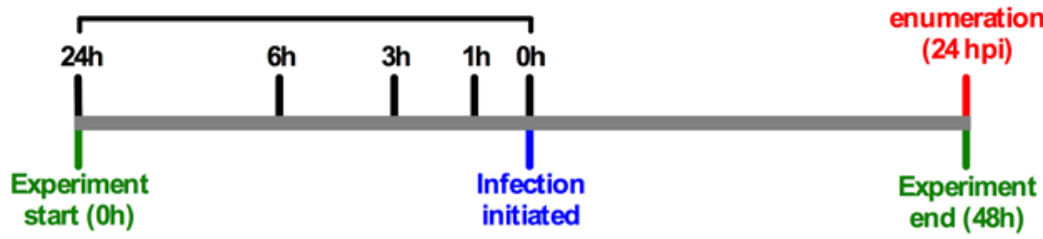

D

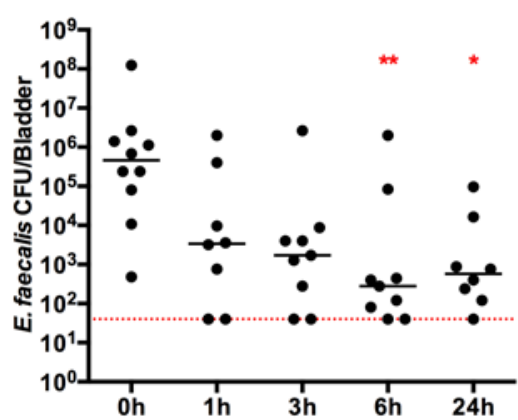

Catheterization time before infection

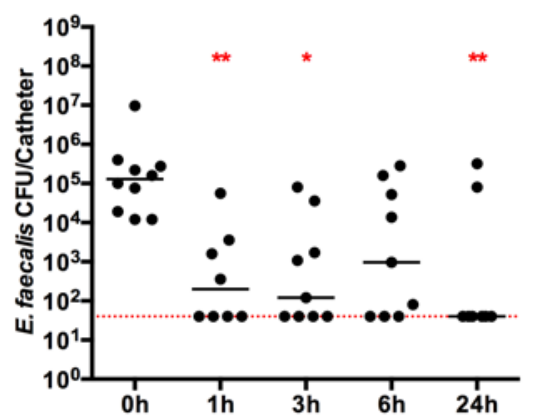

Catheterization time before infection

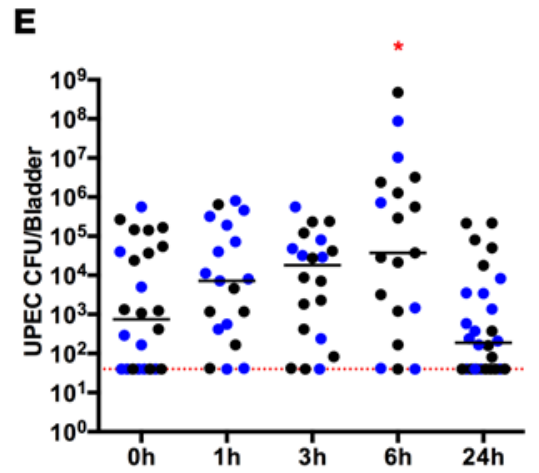

Catheterization time before infection

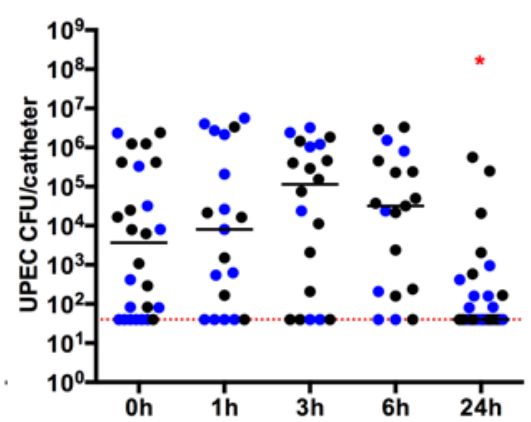

Catheterization time before infection
Figure 3. Susceptibility to infection by uropathogens is altered with increasing time after catheterization. (A and B) Naive female C57BL/6 mice were implanted with catheters and infected with the indicated inocula of $E$. faecalis strain OG1RF or UPEC strain UTI89. Mice were sacrificed 24 hours after infection, and CFU per bladder and per catheter were determined for animals infected with (A) E. faecalis or (B) UPEC. (C) The schematic depicts the experimental approach used in $\mathbf{D}$ and $\mathbf{E}$. Briefly, naive female mice were implanted with catheters and infected at the same time or at the indicated time point after catheterization with $1 \times 10^{4} \mathrm{CFU}$ of $E$. faecalis or UPEC. All animals were sacrificed 24 hours after infection, and CFU per bladder and per catheter were calculated for (D) E. faecalis or (E) UPEC. Each dot represents 1 mouse. Black dots represent animals that were colonized exclusively by the organism inoculated into the bladder. Blue dots indicate animals that were colonized by UPEC and another bacterial species, but the plotted value reflects only the UPEC CFU. The red dotted line represents the lower limit of detection. Experiments were performed 3-5 times ( $n=5-10$ mice per experimental group) and pooled. The presence of the catheter was verified at the time of sacrifice, and mice without a catheter were excluded from the analysis. ${ }^{*} P<0.05$, ${ }^{* *} P<0.01$ for experimental groups compared with the control $0 \mathrm{~h}$ group, Kruskal-Wallis test with Dunn's post-test to correct for multiple comparisons.

Over time, catheterization induced an increase in the total number of cells in the bladder (Figure 2A). To quantify immune cell infiltration, we gated $\mathrm{CD} 45^{+}$immune cells and identified distinct populations based on previously defined protein expression (21) (Figure 2, B-E). Catheterized bladders had increased numbers of $\mathrm{CD}_{4} 5^{+}$cells, which included robust neutrophil accumulation that started at $1 \mathrm{hpc}$ and was statistically significantly increased over the naive cohort from $6 \mathrm{hpc}$ (Figure 2C). Surprisingly, while neutrophil infiltration typically abates after 24 hours (23), in this model neutrophil numbers increased continuously for $168 \mathrm{hpc}$ (7 days). Immature MHC II- monocyte-derived cell infiltration was significantly increased at 24 and $48 \mathrm{hpc}$ compared with naive bladders and approached baseline at 1 week after catheterization (Figure 
A

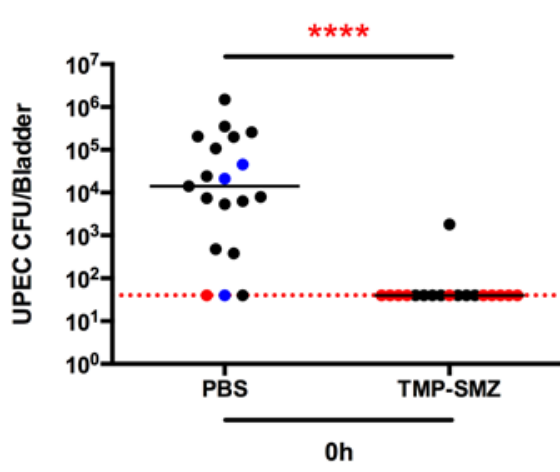

Catheterization time before infection

B

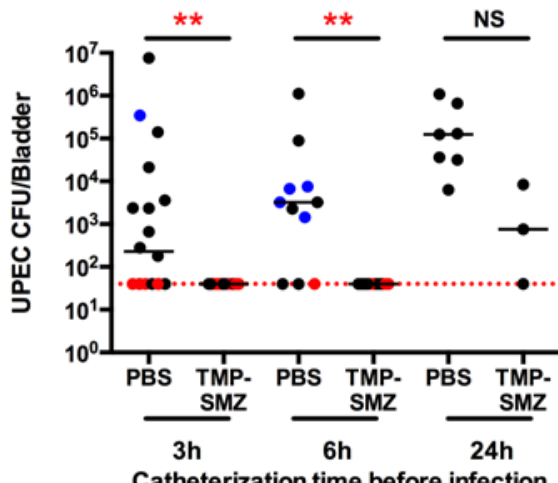

C

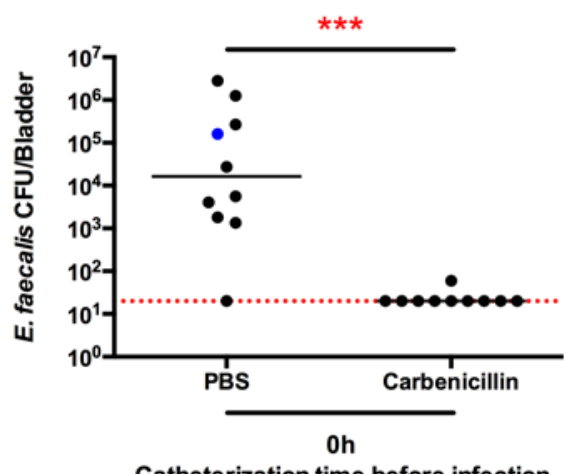

Catheterization time before infection

D

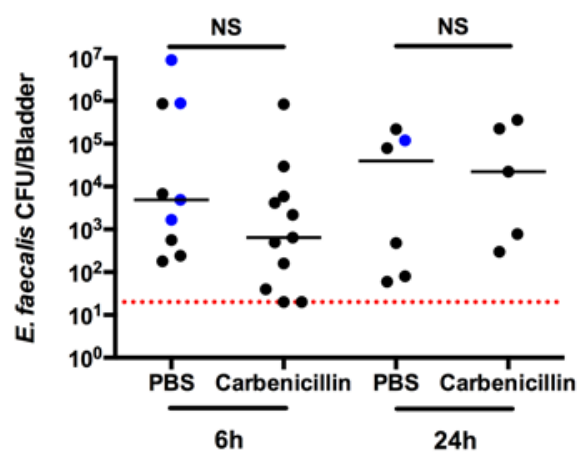

Catheterization time before infection

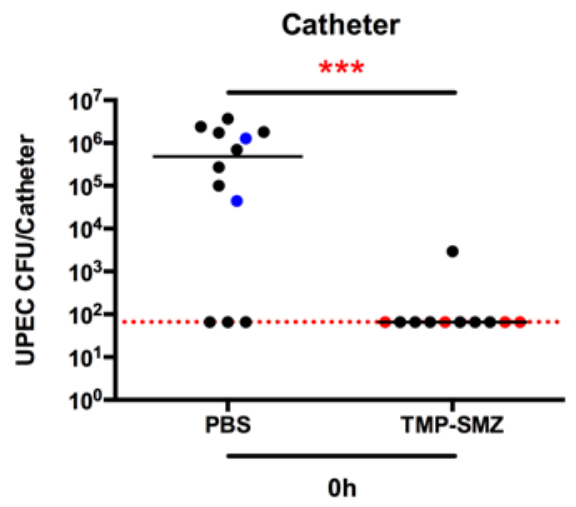

Catheterization time before infection

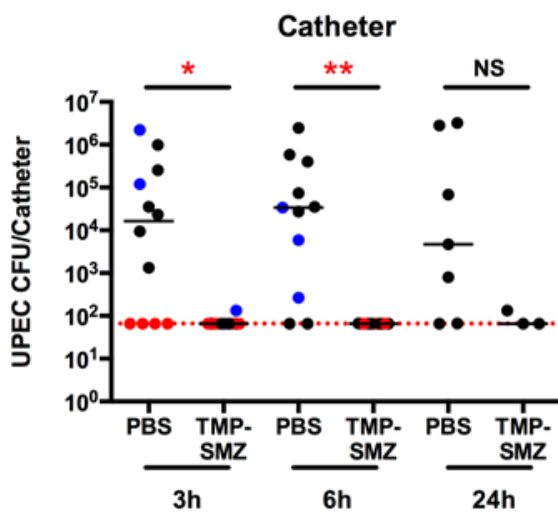

Catheterization time before infection

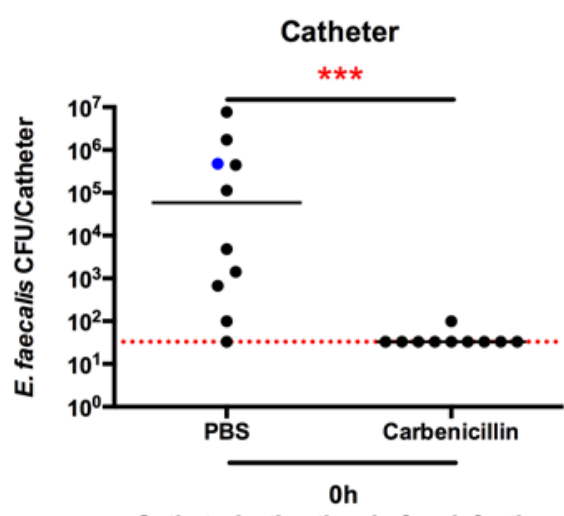

Catheterization time before infection

Catheter

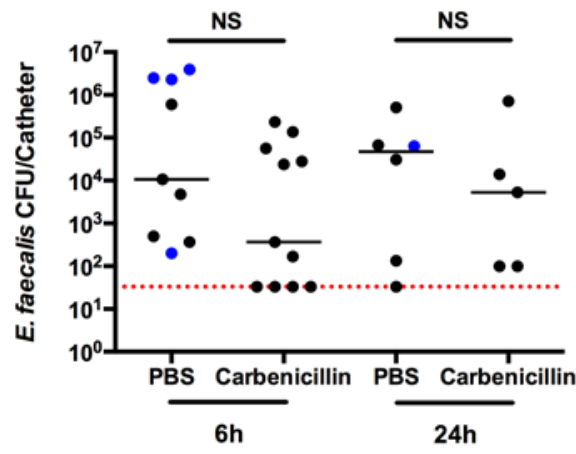

Catheterization time before infection
Figure 4. Antibiotic administration at the time of catheterization prevents infection for 6 hours. Trimethoprim/sulfamethoxazole (TMP-SMZ), carbenicillin, or PBS was administered intraperitoneally to naive female mice concurrent with catheter implantation. Cohorts of animals were then infected with approximately $1 \times 10^{4} \mathrm{CFU}$ of (A and B) UPEC or (C and D) E. faecalis at the same time (A and $\mathbf{C}$ ) or at the indicated time point after catheterization (B and D), CFU per bladder and catheter were assessed 24 hours following infection. Each dot represents 1 mouse, and the experiment was performed 3 times ( $n=7$ mice per experimental group) and pooled. Black dots represent animals that were colonized exclusively by the organism inoculated into the bladder. Red dots represent animals that were negative for UPEC, but positive for an unknown bacterial species. Blue dots indicate animals that were colonized by (A and $\mathbf{B}$ ) UPEC and another bacterial species or by (C and $\mathbf{D})$ E. faecalis and another bacterial species. In both cases, the blue dot reflects the value of UPEC or $E$. faecalis CFU only. The presence of the catheter was verified at the time of sacrifice, and mice without a catheter were excluded from the analysis. ${ }^{*} P<0.05,{ }^{*} P<0.01$ ${ }^{* * *} P<0.001,{ }^{* * * *} P<0.0001$ for comparison of PBS-treated groups to antibiotic-treated groups, Kruskal-Wallis test with Dunn's posttest to correct for multiple comparisons.

2C). Differentiating monocyte-derived cells, defined by expression of Ly6C, upregulation of MHC II, and increased expression of CD64 (Figure 2B) peaked at $24 \mathrm{hpc}$ and remained increased (Figure 2C). Cells characteristic of resident macrophages (CD64 $4^{\text {hi }}$, F4/80 ${ }^{\text {hi }}$, MHC $\mathrm{II}^{+}, \mathrm{CD} 11 \mathrm{~b}^{+}$), which were likely mature monocyte-derived cells, accumulated significantly from 6 to $168 \mathrm{hpc}$ (Figure 2D). Monocytes can also differentiate into DCs in the context of inflammation (24-26). We observed a statistically significant increase in the total number of bladder-associated DCs, comprised of the $\mathrm{CD}_{11 b^{+}}$and $\mathrm{CD} 103^{+} \mathrm{DC}$ subsets, over time from $24 \mathrm{hpc}$ and $6 \mathrm{hpc}$, respectively (Figure 2D). Among nonphagocytic innate immune cells, we noted significant increases over naive bladder populations in the number of NK cells and eosinophils, with a pattern similar to that of neutrophils, in which infiltration increased for the duration of the experiment (Figure 2E). 
To determine the soluble signals mediating immune cell infiltration, we performed multianalyte analysis on supernatants from homogenized noncatheterized bladders or bladders catheterized for 1, 3, 6, and 24 hours. As anticipated from the immune cell infiltration, we observed an increase in cytokines that induce maturation or chemoattraction of neutrophils and monocytes (Figure $2 \mathrm{~F}$ and Supplemental Figure 4). IL-5, IL-12p40, IL-1 $\beta$, CSF3, IL-6, CXCL1, and CCL2 were all significantly increased over naive, noncatheterized animals in at least one time point after catheterization (Supplemental Figure 4). We observed modest nonsignificant changes in expression levels of IL-17, IFN- $\gamma$, CCL5, IL-1 $\alpha$, IL-10, CCL3, TNF- $\alpha$, CCL4, and IL-13. Notably, the expression pattern of significantly increased cytokines did not completely mirror that reported for UPEC infection in the absence of a catheter (27), suggesting that inflammation in the bladder reflects the stimulus to some degree.

Catheter-mediated inflammation differentially affects uropathogen colonization. Despite the prevalence of CAUTI in hospital settings, it seemed counterintuitive that infection can occur in the face of robust inflammation and phagocyte infiltration. As we observed a significant increase in immune infiltrates, starting at $6 \mathrm{hpc}$ and peaking at $24 \mathrm{hpc}$, we hypothesized that catheterized mice would be less susceptible to colonization by uropathogens at time points prior to $24 \mathrm{hpc}$. To test this prediction, we first determined the infectious dose required for colonization in $50 \%\left(\mathrm{ID}_{50}\right)$ and $90 \%\left(\mathrm{ID}_{90}\right)$ of animals for E. faecalis strain OG1RF and UPEC strain UTI89 in the CAUTI mouse model. For E. faecalis, the $\mathrm{ID}_{50}$ for bladder and catheter was approximately $10^{3} \mathrm{CFU}$ and the $\mathrm{ID}_{90}$ was approximately $10^{4} \mathrm{CFU}$ (Figure $3 \mathrm{~A}$ ). For UPEC, the $\mathrm{ID}_{50}$ was less than $10^{3} \mathrm{CFU}$ for bladder and $10^{3} \mathrm{CFU}$ for catheter; the $\mathrm{ID}_{90}$ was approximately $10^{3} \mathrm{CFU}$ for bladder and $10^{4} \mathrm{CFU}$ for catheter (Figure 3B). For this and all subsequent analyses, animals in which no bacterial growth was detected in the bladder or on the catheter following infection are plotted at the limit of detection of the assay, denoted by a red dotted lines in graphs representing CFU (Figure 3, Figure 4, Supplemental Figure 5, Supplemental Figure 7, and Supplemental Figure 8). It is important to note that these $\mathrm{ID}_{90}$ doses do not result in colonization in noncatheterized animals (Supplemental Figure 5 and refs. 28, 29).

We infected mice at the same time as catheter implantation, or at defined hpc, with $10^{4} \mathrm{CFU}$ of $E$. faecalis or UPEC (Figure 3C). Following E. faecalis infection concurrent with catheter implantation, we recovered $4.6 \times 10^{5} \mathrm{CFU} /$ bladder and $1.3 \times 10^{5} \mathrm{CFU} /$ catheter at 24 hours after infection (Figure $3 \mathrm{D}, 0 \mathrm{~h}$ ). However, when infection was initiated 1, 3, 6, or 24 hours after catheter implantation, significantly fewer bladder- and catheter-associated bacteria were observed after a 24 hour infection (Figure 3D). UPEC infection concomitant with catheter implantation, gave rise to $7.5 \times 10^{2} \mathrm{CFU}$ per bladder and $3.7 \times 10^{3} \mathrm{CFU}$ per catheter (Figure 3E, 0h). CFU following UPEC infection initiated at 1 or $3 \mathrm{hpc}$ increased moderately in bladders or on catheters; however, CFU were significantly increased in the bladder when infection was initiated 6 hpc. Finally, a significant decrease in catheter-associated UPEC was observed when infection was initiated $24 \mathrm{hpc}$ (Figure 3E). We occasionally noted the presence of other bacterial species in addition to the infecting UPEC strain (Figure 3E, blue dots, and Supplemental Figure 6A), but this did not affect the mean UPEC titer.

Prophylactic antibiotic treatment prevents CAUTI. Our results suggested that the inflammation induced by catheterization created an increased risk for UPEC infection in the first 6 hours and for E. faecalis at the time of catheterization. To test whether catheter-mediated bacterial colonization could be prevented, we catheterized animals and treated cohorts with trimethoprim/sulfamethoxazole (TMP-SMZ), an antibiotic combination commonly used to treat UTI (30). Mice received a single high-dose ( $2 \mathrm{mg} / \mathrm{mouse})$ intraperitoneal injection of antibiotic at the time of catheter implantation, or PBS injection as a control, and were subsequently infected with $10^{4} \mathrm{CFU}$ of UPEC at $0,3,6$, or $24 \mathrm{hpc}$. Qualitative assessment of infection by urine culture was measured just prior to sacrifice, and no bacteria were observed in antibiotic-treated mice when infection was initiated in the first 6 hours after catheterization (Supplemental Figure 7). We observed that a single antibiotic treatment prevented colonization of the bladder and catheters when infection was initiated at the same time as catheterization, a time point modeling health care worker-induced infection (Figure 4A). When infection was initiated at 3, 6, or $24 \mathrm{hpc}$, to simulate bacterial ascension of the catheter, UPEC colonization of bladders and catheters was almost entirely eliminated up to 6 hpc (Figure 4B). While UPEC were nearly completely absent from antibiotic-treated mice until $6 \mathrm{hpc}$, we occasionally observed the presence of other bacterial species in UPEC-colonized mice, similar to that observed in experiments depicted in Figure 3 (Figure 4, A and B, red dots, and Supplemental Figure 6). 16S rRNA sequence analysis revealed that Staphylococcus lentus, a mouse commensal (31), as well as Staphylococci identified as either Staphylococcus xylosus (a mouse commensal, ref. 32) or Staphylococcus saprophyticus (a human uro- 
pathogen not previously reported in mice, ref. 33) contributed to the polymicrobial colonization of these animals. Prophylactic prevention of UPEC CAUTI prompted us to consider whether an antibiotic targeting Gram-positive species may serve to further protect animals against bacterial colonization.

Notably, E. faecalis strain OG1RF was resistant to killing by TMP-SMZ, and colonization was not prevented in mice treated with TMP-SMZ and subsequently infected with E. faecalis (Supplemental Figure 8). This same strain was sensitive to carbenicillin, an antibiotic related to penicillin used to treat UTI caused by Enterococcus (34). Using a similar experimental scheme, we administered a single injection of carbenicillin at $2 \mathrm{mg}$ /mouse, or PBS as a control, concurrent with catheter implantation. Mice were infected with $10^{4}$ CFU of E. faecalis at 0,6 , or $24 \mathrm{hpc}$. We observed that, similar to UPEC infection treated with TMP-SMZ, $E$. faecalis colonization was inhibited by a single antibiotic treatment at the time of catheter implantation (Figure 4C). Notably, however, colonization was reduced but not prevented when infection was initiated 6 or 24 hpc (Figure 4D).

\section{Discussion}

The primary purpose of this study was to investigate the impact of catheterization on host immune response and susceptibility to uropathogen infection. Catheterization induces physical changes in the bladder and provides a structural surface permitting colonization by uropathogens. In animal studies, infected and noninfected catheterized bladders show profound tissue damage, including disruption of the epithelial barrier, as compared with noncatheterized infected rodents $(14,35)$. Our studies have revealed that the initial inflammatory host response to catheterization is sustained for at least 1 week, as evidenced by continuous neutrophil recruitment to the catheterized bladder. These findings suggest that long-term catheterization potently induces chronic inflammation of the bladder, which likely results in permanent structural changes $(11,12,36)$.

Herein, we have expanded our understanding of the impact of catheterization on host susceptibility to bacterial colonization. Our findings are in line with previous reports demonstrating that CFU recovered from experimental E. faecalis and Pseudomonas aeruginosa infections are higher in catheterized animals as compared with noncatheterized animals $(14,35,37)$. Indeed, that the $\mathrm{ID}_{90}$ for E. faecalis and UPEC are greatly reduced in catheter-bearing animals compared with noncatheterized mice highlights the truly vulnerable host state induced by catheter implantation. In addition, we observed that $E$. faecalis colonization of precatheterized bladders was less efficient than infection at the time of catheter implantation, whereas UPEC was able to efficiently colonize the bladder up to $24 \mathrm{hpc}$. These data suggest that bladder catheterization and the associated inflammation differentially affect uropathogen infection after implantation, at or before the peak of myeloid cell infiltration at 6-24 hpc. By contrast, previous studies using an E. faecalis inoculum 3 logs greater than the $\mathrm{ID}_{90}$ reported here, found that infection at the time of catheterization was similar to colonization at $24 \mathrm{hpc}(13)$, suggesting that the greater infectious dose may overwhelm the host response, eliminating the protection against infection that we observed here.

Additionally, in the course of our experiments, we observed the presence of other bacterial species in some but not all bladders and catheters, i.e., polymicrobial colonization. Notably, our experimental analysis of bacterial load did not rely upon antibiotic selection for the infecting strain, which permitted us to identify monomicrobially and polymicrobially infected mice. Importantly, placement of a catheter in the absence of bacterial inoculation did not result in bacterial outgrowth from bladders or catheters when plated on agar, demonstrating that the presence of additional bacterial species is likely not due to contamination in the course of experimentation. Indeed, we speculate that the combined influence of catheterization and infection creates a niche for polymicrobial colonization of the bladder by commensal species, such as the mouse commensals $S$. xylosus and $S$. lentus isolated in this study, or other opportunistic bacterial species. These results may also reflect the varying commensal population in commercially available mice, as while the overall frequency of colonization of non-E. faecalis and non-UPEC species was never higher than the inoculated organism, the experiment-to-experiment frequency of polymicrobial colonization was highly varied. These findings likely reflect the true nature of CAUTI in humans as well.

Notably, published studies have assessed catheter and bladder colonization following infection at the same time as catheterization (13). While this model simulates infection induced by insertion of the medical device, it cannot be used to explore outcomes of bacterial ascension after catheterization. Moreover, in previous studies, the infecting bacterial doses far exceeded the $\mathrm{ID}_{90}$ for CAUTI that we determined here. In this study, we assessed the relative susceptibility to infection either at the same time as catheterization or at 
early time points following implantation. We observed that within the first 24 hours of catheterization, susceptibility to $E$. faecalis infection diminished concurrently with the onset of innate immune cell infiltration, whereas UPEC retained the capacity to colonize the host despite robust infiltration until $24 \mathrm{hpc}$.

Treatment with mannoside compounds, which block the binding of UPEC to the bladder surface and prevent colonization, effectively limited implant and bladder colonization in a model of UPEC CAUTI when delivered intraperitoneally 30 minutes prior to catheterization (38). In addition, mannoside compounds acted synergistically with TMP-SMZ to further reduce the level of CFU recovered from catheters and bladders 6 hours after infection (38). Unfortunately, these compounds, despite their efficacy in preclinical models, remain to be tested in clinical trials and are not currently available. Indeed, at this point in time, therapeutic strategies to treat UTI and CAUTI rely primarily on antibiotics, including daily low-dose prophylaxis to prevent infection in patients prone to recurrence (30). Reliance on antibiotic therapy is especially concerning given the rise in multidrug antibiotic resistance in common UPEC and other uropathogen strains (39). In addition, the impact on the host microbiota cannot be discounted in the context of continuous low-dose antibiotic treatment. In real world conditions (e.g., emergency, surgery, sudden illness), a patient would not have the opportunity for days of prophylactic treatment. In this study design, we considered the relative advantages and disadvantages of antibiotic treatment as a prophylactic measure to limit CAUTI. One of the benefits of the use of TMP-SMZ and carbenicillin is their short half-lives. TMP-SMZ persists for less than 1 day in adults and 5 hours in rodents $(40,41)$, whereas carbenicillin has a half-life of less than 3 hours in the serum but concentrates in the urine of patients before it is eliminated (42). These short half-lives may limit selective pressure on uropathogens to acquire antibiotic resistance. Using this approach, we effectively eliminated colonization of bladders and implanted catheters in animals infected up to 6 hours after catheter implantation, which corresponds with the observed window of vulnerability.

These findings provide a rational basis for our proposed clinical intervention of a single high-dose antibiotic treatment at the time of catheter placement. Indeed, given the recent report that simple preventative measures, such as glove changing and hand washing, reduce the incidence of UTI in long-term indwelling catheter patients (43), our results strongly suggest that these measures plus the administration of acute prophylactic therapy should reduce the incidence of UTI in all patients undergoing catheterization. Our proposed therapeutic intervention will also directly affect health care providers, as CAUTI complicates care and worsens outcomes in individuals that are already sick or injured. CAUTI exerts a particularly high economic burden on the health care system as the most common hospital-associated infection $(9,10)$ and disproportionately affects the aging patient population (44). The impact of such a treatment has profound potential when considering the cost/benefit ratio of short-term prophylactic therapy versus the inflated cost of treating nosocomial CAUTI after catheterization. Preventing bacteriuria and symptomatic infection will decrease the need for additional intervention, improve patient outcomes, and directly lead to health care system cost savings through reduced rates of infection and development of complications.

\section{Methods}

Bacterial strains. The human UPEC cystitis isolate UTI89 $(45,46)$ and the fluorescent protein-expressing strain UTI89-RFP (21) were grown overnight in static cultures at $37^{\circ} \mathrm{C}$ in Luria-Bertani broth (LB) in the presence of antibiotic ( $50 \mu \mathrm{g} / \mathrm{ml}$ kanamycin) where appropriate. UTI89 is sensitive to antibiotics, and UTI89-RFP is resistant to kanamycin. The E. faecalis strain OG1RF (47) was grown statically in brain heart infusion (BHI) broth or agar at $37^{\circ} \mathrm{C}$ overnight in the presence of fusidic acid $(25 \mu \mathrm{g} / \mathrm{ml})$ or rifampin $(25 \mu \mathrm{g} / \mathrm{ml})$.

Catheterization and bacterial infections. Implantation of catheters was performed as previously described (14). Briefly, female C57BL/6 mice, 6-8 weeks of age, from Charles River France or InVivos Singapore were anesthetized with $100 \mathrm{mg} / \mathrm{kg}$ ketamine and $5 \mathrm{mg} / \mathrm{kg}$ xylazine or isoflurane (4\%), respectively, catheterized, and subsequently infected with $10^{3}, 10^{4}$, or $10^{5} \mathrm{CFU}$ of E. faecalis or UPEC in $50 \mu 1 \mathrm{PBS}$ via a catheter introduced into the urethra. Animals were sacrificed either by cervical dislocation or carbon dioxide inhalation. To calculate CFU, bladders were aseptically removed and homogenized in $1 \mathrm{ml}$ PBS. Catheters removed from the bladders were vortexed and sonicated in $1 \mathrm{ml}$ PBS. Serial dilutions were plated on MacConkey agar or BHI agar supplemented with $10 \mu \mathrm{g} / \mathrm{ml}$ colistin and $10 \mu \mathrm{g} / \mathrm{ml}$ nalidixic acid to identify UPEC or E. faecalis, respectively. To determine whether bacterial species were present in addition to the inoculated UPEC or E. faecalis, serial dilutions were also plated on LB and BHI. Non-E. faecalis and non-UPEC species were identified by $16 \mathrm{~S}$ rDNA sequencing using primers $27 \mathrm{~F}$ ( $5^{\prime}$-AGAGTTTGATYMTGGCTCAG-3') and 1492R (5'-TACGGYTACCTTGTTACGACTT-3'). Animals without catheters at the 
time of sacrifice were not included in the analyses. This was more common in animals catheterized for longer time periods.

$R N A$ sequencing. Prior to RNA extraction, bladders were aseptically removed and incubated overnight in RNAlater (Qiagen) before storage at $-80^{\circ} \mathrm{C}$. Bladders were transferred to $2-\mathrm{ml}$ homogenization tubes containing 1.4-mm ceramic spheres (MPBio) and $1 \mathrm{ml}$ Trizol (Life Technologies). Bladders were then homogenized on a FastPrep-24 tissue grinder (MPBio) by 3 grindings of 60 seconds at $6 \mathrm{~m} / \mathrm{s}$ with 5 minutes rest on ice between grinding. RNA was isolated according to the manufacturer's protocols. Contaminating DNA was removed using the Ambion TURBO DNA-free Kit (Life Technologies) prior to rRNA depletion using a Ribo-zero gold rRNA removal kit (Illumina). RNA and DNA concentrations were quantified using the Qubit RNA HS assay kit and the Qubit dsDNA HS assay kit, respectively (Life Technologies). We constructed one sequencing library from each replicate sample. Multiplexed sequencing libraries were generated from 50-200 ng rRNA-depleted RNA (per library) using the Illumina TruSeq v3 protocol, and each library was tagged with one barcode from the Illumina TruSeq LT NA barcode collection to permit library pooling. Library concentration was standardized to $2 \mathrm{nM}$ and confirmed using qPCR on a ViiA-7-real-time thermocycler (Applied Biosystems). Libraries were pooled at equal volumes and sequenced on 2 lanes of an Illumina Hiseq 2500 v.2 (Illumina) rapid run, with a paired-end read length of $150 \mathrm{bp}$.

Processing and analysis of RNA sequencing data. The reads were quality checked and adaptor removed using cutadapt-1.4.1 with default parameters. Filtered reads were mapped to the mm10 mouse genome using tophat-2.0.11.Linux_x86_64 (48) using default parameters, and read counts per transcriptional unit were calculated using HTSeq-0.6.1 (49) with default parameters (except "-stranded" was set to "no"). In the resultant raw read count matrix, genes (rows) were indexed with NCBI Refseq identifiers and samples were indexed in columns. We studied differential expression between catheterized and naive animals using the statistical models implemented in the R/Bioconductor package DESeq2 (version 1.10.1); specifically, we used default settings in the DESeq and results functions. For further analysis, we converted Refseq identifiers to Entrez Gene identifiers using the NCBI gene2refseq (downloaded 03/03/2016). In cases in which multiple Refseq identifiers were associated with one Entrez identifier, we generated a one-to-one mapping by selecting the Refseq identifier with the highest mean expression across all 6 samples. For comparison to ImmGen data, we replaced Entrez Gene identifiers with their corresponding official gene symbols.

Functional analysis of gene expression data. The following analyses were performed in R 3.2.2 (50). We sourced expression data from ImmGen by downloading all 681 CEL files archived in NCBI GEO (accession no. GSE15907) (19) and processed them using the RMA method implemented in the R/Bioconductor package oligo (version 1.34.0). We annotated Affymetrix probe set identifiers to mouse gene symbols using the $\mathrm{R} /$ Bioconductor package mogene10sttranscriptcluster. $d b$ (version 8.4.0). Representative expression profiles for each cell type were sourced from Supplemental Table 2 of Jojic et al. (19), resulting in 327 expression profiles from between 14 and 64 representative samples in each of 12 broad categories of immune cell. We also sourced cell-type selective or specific modules of genes, as defined by ImmGen, and examined whether these genes had higher expression levels observable in catheterized animals relative to naive animals. For each gene set (defined using the coarse module classification analysis of Jojic et al., ref. 19) we tested whether the mean log fold change in expression between catheterized and noncatheterized animals was different from 0 using a 1-sample $t$ test, with the minimum gene number per module was set to 3 (79 of 81 modules included), and we corrected for the number of gene sets tested using the Bonferroni procedure. For each category of immune cell type, we also calculated the enrichment score using the method of Benita et al. (51) and examined the number of top-ranked differentially expressed genes upregulated in catheterized mice compared with naive mice that fell in the top $1 \%$ of the distribution of scores for each cell type and compared these with equal sized cohorts of randomly selected genes (Supplemental Table 5).

We used the GO biological process provided in the R/Bioconductor package GO.db (version 3.2.2) and sourced annotations from the gene2go file available from the NCBI Gene database (downloaded 03/03/2016). The ontology and annotations were processed using a modified version of the R/Bioconductor package ontoTools (version 1.28.0) (52). We tested the hypothesis that differentially expressed genes would be associated with specific biological processes by constructing 2-by-2 contingency tables for each included GO biological process term by categorizing genes as being either differentially expressed or not and as being annotated to the term or not. We tested for random assignment among these 4 possible categories with Fisher's exact test (53), correcting the raw $P$ values for the number of terms tested using the Benjamini-Hochberg correction (54). Rather than testing all GO terms, we filtered terms using their information content (IC) (55), 
a dimensionless number associated with the frequency of occurrence of a given term in the complete set of detected genes (very general terms will have a small IC value and very specific terms will have a high IC value). We selected terms that have an IC value of between 3 and 4, resulting in a set of 157 included terms that represent an appropriate trade-off between the total number of included terms and specificity of functional insight. Analyses were performed independently, with differentially expressed gene lists identified from each experiment ( 2 experiments, $n=3$ animals/experimental group). The entire $\mathrm{R}$ workflow and input data files are available online (https://github.com/rbhwilliams/JCI-Insights-paper-data-and-analysis).

Spectral analysis of bladder tissue. Bladders were prepared as previously described (21). At indicated time points, bladders were removed, catheters were recovered, and tissue was minced into digestion buffer containing $0.34 \mathrm{U} / \mathrm{ml}$ of Liberase TM (Roche) in PBS at $4^{\circ} \mathrm{C}$. Minced tissue was incubated at $37^{\circ} \mathrm{C}$ for 1 hour, with vigorous shaking every 15 minutes. Digestion was arrested by the addition of PBS supplemented with $2 \%$ FBS and $0.2 \mu \mathrm{M}$ EDTA and passed through $100-\mu \mathrm{m}$ cell strainers. Following a 10 -minute incubation in Fc block (BD Biosciences), cells were immunolabeled with the antibodies listed in Supplemental Table 6. Cells were acquired on a Sony SP6800 3-laser spectral analyzer. Spectral data were unmixed using the WLSM algorithm. Total cell counts in the bladder were determined on a BD LSRFortessa cytometer (using DIVA software and analyzed by FlowJo software) by the addition of AccuCheck Counting beads (Invitrogen) to a known volume of sample after immunolabeling, just prior to acquisition.

Luminex MAP analysis. Naive and/or catheterized bladders were removed the indicated time points and homogenized in $1 \mathrm{ml}$ PBS on ice. A $100-\mu 1$ aliquot was removed to confirm that bladders were sterile by plating on bacterial agar, prior to clarification by microcentrifugation $\left(13,000 \mathrm{~g}, 4^{\circ} \mathrm{C}, 5\right.$ minutes $)$. Supernatants were stored at $-80^{\circ} \mathrm{C}$ until assessment by the Bio-Plex Pro mouse cytokine 23-plex assay kit (Bio-Rad Laboratories), according to the manufacturer's recommendations (27). Just prior to analysis, after thawing, samples were centrifuged a second time to remove any remaining cell debris. All samples were assessed using the same kit lot and at the same time to avoid interassay variability.

Sequencing data availability. Sequencing data are available at NCBI's BioProject (accession no. PRJNA335539).

Statistics. Statistical analysis pertaining to RNA sequencing data (Figure 1 and Supplemental Figure 1-3) is described above. GraphPad Prism was used to evaluate statistical significance $(P<0.05)$ in Figures $2-4$ and Supplemental Figure 4). Horizontal bars in graphs depict medians and statistical significance was determined by nonparametric Kruskal-Wallis tests. Multiple comparisons were made by comparing each column with the control naive column or by pairwise comparison with Dunn's post-test to correct for multiple comparisons, as indicated in the figure legends. $P$ values of less than 0.05 were considered significant.

Study approval. At Institut Pasteur, mouse experiments were conducted with approval of the Comité d'éthique en expérimentation animale Paris Centre et Sud (protocol no. 2016-0010) in application of the European Directive 2010/63/EU. At Nanyang Technological University, mouse experiments were performed with ethical approval by the ARF-SBS/NIE Nanyang Technological University Institutional Animal Care and Use Committee under protocol ARF-SBS/NIE-A0247.

\section{Author contributions}

HMSG, MR, and SH conceptualized and performed experiments, analyzed data, and critically revised the manuscript; MLA critically reviewed data and the manuscript; RBHW conceptualized experiments, analyzed data, and wrote the manuscript; MAI and KAK conceptualized and performed experiments, analyzed data, and wrote the manuscript.

\section{Acknowledgments}

We gratefully acknowledge insightful discussions with and critical reading of the manuscript by Pascale Guiton and Darragh Duffy. We are deeply indebted to Koji Futamura for his assistance in establishing parameters for spectral analysis and providing valuable input. We thank the Flow Cytometry Facility of the Pasteur Institute (Cytometry Platform/Center for Translational Research) for providing training and assistance. We also thank Adeline Yong and Kelvin Chong for their assistance in animal experiments, Krithika Arumugam for her assistance with sequence data processing, and Magnus Fontes for discussion of data analysis. This work benefitted from data assembled by the ImmGen Consortium (20). The authors wish to acknowledge the following funding: HMSG, SH, RBHW, and KAK were supported by the National Research Foundation (NRF) and Ministry of Education Singapore under its Research 
Centre of Excellence Programme. HMSG, SH, and KAK were supported by the NRF under its Singapore NRF Fellowship program (NRF-NRFF2011-11) and by the Ministry of Education Singapore under its Tier 2 program (MOE2014-T2-1-129). MAI was supported by funding from the European Union Seventh Framework Programme Marie Curie Action (PCIG11-GA-2012-3221170). Finally, this project was realized through the Merlion Programme, sponsored by the French Ministry of Foreign Affairs and Nanyang Technological University, to KAK and MAI.

Address correspondence to: Molly A. Ingersoll, Institut Pasteur, 25 Rue du Docteur Roux, 75015 Paris, France. Phone: 33.145.68.80.71; E-mail: molly.ingersoll@pasteur.fr. Or to: Kimberly A. Kline, Singapore Centre on Environmental Life Sciences Engineering, 60 Nanyang Drive, Singapore 637551. Phone: 65.9755.3601; E-mail: kkline@ntu.edu.sg.

MLA's present address is: Department of Cancer Immunology, Genentech Inc., San Francisco, California, USA.

1. Foxman B. The epidemiology of urinary tract infection. Nat Rev Urol. 2010;7(12):653-660.

2. Hartstein AI, Garber SB, Ward TT, Jones SR, Morthland VH. Nosocomial urinary tract infection: a prospective evaluation of 108 catheterized patients. Infect Control. 1981;2(5):380-386.

3. Hooton TM, et al. Diagnosis, prevention, and treatment of catheter-associated urinary tract infection in adults: 2009 International Clinical Practice Guidelines from the Infectious Diseases Society of America. Clin Infect Dis. 2010;50(5):625-663.

4. Saint S, et al. Preventing hospital-acquired urinary tract infection in the United States: a national study. Clin Infect Dis. 2008;46(2):243-250.

5. Saint S. Clinical and economic consequences of nosocomial catheter-related bacteriuria. Am J Infect Control. 2000;28(1):68-75.

6. Platt R, Polk BF, Murdock B, Rosner B. Mortality associated with nosocomial urinary-tract infection. N Engl J Med. 1982;307(11):637-642.

7. Givens CD, Wenzel RP. Catheter-associated urinary tract infections in surgical patients: a controlled study on the excess morbidity and costs. J Urol. 1980;124(5):646-648.

8. Tambyah PA, Knasinski V, Maki DG. The direct costs of nosocomial catheter-associated urinary tract infection in the era of managed care. Infect Control Hosp Epidemiol. 2002;23(1):27-31.

9. Klevens RM, et al. Estimating health care-associated infections and deaths in U.S. hospitals, 2002. Public Health Rep. 2007;122(2):160-166.

10. Tambyah PA, Knasinski V, Maki DG. The direct costs of nosocomial catheter-associated urinary tract infection in the era of managed care. Infect Control Hosp Epidemiol. 2002;23(1):27-31.

11. Peychl L, Zalud R. [Changes in the urinary bladder caused by short-term permanent catheter insertion]. Cas Lek Cesk. 2008;147(6):325-329.

12. Delnay KM, Stonehill WH, Goldman H, Jukkola AF, Dmochowski RR. Bladder histological changes associated with chronic indwelling urinary catheter. J Urol. 1999;161(4):1106-1108; discussion 1108.

13. Guiton PS, Hannan TJ, Ford B, Caparon MG, Hultgren SJ. Enterococcus faecalis overcomes foreign body-mediated inflammation to establish urinary tract infections. Infect Immun. 2013;81(1):329-339.

14. Guiton PS, Hung CS, Hancock LE, Caparon MG, Hultgren SJ. Enterococcal biofilm formation and virulence in an optimized murine model of foreign body-associated urinary tract infections. Infect Immun. 2010;78(10):4166-4175.

15. Flores-Mireles AL, Walker JN, Caparon M, Hultgren SJ. Urinary tract infections: epidemiology, mechanisms of infection and treatment options. Nat Rev Microbiol. 2015;13(5):269-284.

16. Maki DG, Tambyah PA. Engineering out the risk for infection with urinary catheters. Emerging Infect Dis. 2001;7(2):342-347.

17. Flores-Mireles AL, Pinkner JS, Caparon MG, Hultgren SJ. EbpA vaccine antibodies block binding of Enterococcus faecalis to fibrinogen to prevent catheter-associated bladder infection in mice. Sci Transl Med. 2014;6(254):254ra127.

18. Rock KL, Latz E, Ontiveros F, Kono H. The sterile inflammatory response. Annu Rev Immunol. 2010;28:321-342.

19. Jojic V, et al. Identification of transcriptional regulators in the mouse immune system. Nat Immunol. 2013;14(6):633-643.

20. Heng TS, Painter MW, Immunological Genome Project Consortium. The Immunological Genome Project: networks of gene expression in immune cells. Nat Immunol. 2008;9(10):1091-1094.

21. Mora-Bau G, Platt AM, van Rooijen N, Randolph GJ, Albert ML, Ingersoll MA. Macrophages subvert adaptive immunity to urinary tract infection. PLoS Pathog. 2015;11(7):e1005044.

22. Futamura K, et al. Novel full-spectral flow cytometry with multiple spectrally-adjacent fluorescent proteins and fluorochromes and visualization of in vivo cellular movement. Cytometry A. 2015;87(9):830-842.

23. Nathan C. Points of control in inflammation. Nature. 2002;420(6917):846-852.

24. Randolph GJ, Inaba K, Robbiani DF, Steinman RM, Muller WA. Differentiation of phagocytic monocytes into lymph node dendritic cells in vivo. Immunity. 1999;11(6):753-761.

25. Varol C, et al. Monocytes give rise to mucosal, but not splenic, conventional dendritic cells. J Exp Med. 2007;204(1):171-180.

26. Ginhoux F, et al. Langerhans cells arise from monocytes in vivo. Nat Immunol. 2006;7(3):265-273.

27. Ingersoll MA, Kline KA, Nielsen HV, Hultgren SJ. G-CSF induction early in uropathogenic Escherichia coli infection of the urinary tract modulates host immunity. Cell Microbiol. 2008;10(12):2568-2578.

28. Rosen DA, Hung CS, Kline KA, Hultgren SJ. Streptozocin-induced diabetic mouse model of urinary tract infection. Infect Immun. 2008;76(9):4290-4298. 
29. Kau AL, Martin SM, Lyon W, Hayes E, Caparon MG, Hultgren SJ. Enterococcus faecalis tropism for the kidneys in the urinary tract of C57BL/6J mice. Infect Immun. 2005;73(4):2461-2468.

30. Barber AE, Norton JP, Spivak AM, Mulvey MA. Urinary tract infections: current and emerging management strategies. Clin Infect Dis. 2013;57(5):719-724.

31. Tavakkol Z, et al. Resident bacterial flora in the skin of C57BL/6 mice housed under SPF conditions. J Am Assoc Lab Anim Sci. 2010;49(5):588-591.

32. Gozalo AS, Hoffmann VJ, Brinster LR, Elkins WR, Ding L, Holland SM. Spontaneous Staphylococcus xylosus infection in mice deficient in NADPH oxidase and comparison with other laboratory mouse strains. J Am Assoc Lab Anim Sci. 2010;49(4):480486.

33. Kline KA, et al. Characterization of a novel murine model of Staphylococcus saprophyticus urinary tract infection reveals roles for Ssp and SdrI in virulence. Infect Immun. 2010;78(5):1943-1951.

34. Fiedelman W. Carbenicillin therapy of urinary tract infections due to difficult uropathogens. III. Enterococcus. Curr Ther Res Clin Exp. 1975;18(1 s supp1):249-256.

35. Kurosaka Y, Ishida Y, Yamamura E, Takase H, Otani T, Kumon H. A non-surgical rat model of foreign body-associated urinary tract infection with Pseudomonas aeruginosa. Microbiol Immunol. 2001;45(1):9-15.

36. Vaidyanathan S, Soni BM, Bingley J, Brown E, Markey S. Prevention of pressure sore caused by indwelling urinary catheters. Spinal Cord. 2002;40(9):489; author reply 490.

37. Kadurugamuwa JL, Modi K, Yu J, Francis KP, Purchio T, Contag PR. Noninvasive biophotonic imaging for monitoring of catheter-associated urinary tract infections and therapy in mice. Infect Immun. 2005;73(7):3878-3887.

38. Guiton PS, et al. Combinatorial small-molecule therapy prevents uropathogenic Escherichia coli catheter-associated urinary tract infections in mice. Antimicrob Agents Chemother. 2012;56(9):4738-4745.

39. Zowawi HM, et al. The emerging threat of multidrug-resistant Gram-negative bacteria in urology. Nat Rev Urol. 2015;12(10):570-584.

40. Singlas E, et al. Pharmacokinetics of sulfamethoxazole--trimethoprim combination during chronic peritoneal dialysis: effect of peritonitis. Eur J Clin Pharmacol. 1982;21(5):409-415.

41. Bartlett MS, Shaw MM, Smith JW, Meshnick SR. Efficacy of sulfamethoxypyridazine in a murine model of Pneumocystis carinii pneumonia. Antimicrob Agents Chemother. 1998;42(4):934-935.

42. Smith CB, Finland M. Carbenicillin: activity in vitro and absorption and excretion in normal young men. Appl Microbiol. 1968;16(11):1753-1760.

43. Schelling K, et al. Reducing catheter-associated urinary tract infections in a neuro-spine intensive care unit. Am J Infect Control. 2015;43(8):892-894.

44. Kline KA, Bowdish DM. Infection in an aging population. Curr Opin Microbiol. 2016;29:63-67.

45. Chen SL, et al. Identification of genes subject to positive selection in uropathogenic strains of Escherichia coli: a comparative genomics approach. Proc Natl Acad Sci U S A. 2006;103(15):5977-5982.

46. Mulvey MA, Schilling JD, Hultgren SJ. Establishment of a persistent Escherichia coli reservoir during the acute phase of a bladder infection. Infect Immun. 2001;69(7):4572-4579.

47. Dunny GM, Brown BL, Clewell DB. Induced cell aggregation and mating in Streptococcus faecalis: evidence for a bacterial sex pheromone. Proc Natl Acad Sci U S A. 1978;75(7):3479-3483

48. Kim D, Pertea G, Trapnell C, Pimentel H, Kelley R, Salzberg SL. TopHat2: accurate alignment of transcriptomes in the presence of insertions, deletions and gene fusions. Genome Biol. 2013;14(4):R36.

49. Anders S, Pyl PT, Huber W. HTSeq--a Python framework to work with high-throughput sequencing data. Bioinformatics. 2015;31(2):166-169.

50. R Development Core Team. R Project for Statistical Computing. https://www.r-project.org. Accessed August 24, 2016.

51. Benita Y, Cao Z, Giallourakis C, Li C, Gardet A, Xavier RJ. Gene enrichment profiles reveal T-cell development, differentiation, and lineage-specific transcription factors including ZBTB25 as a novel NF-AT repressor. Blood. 2010;115(26):5376-5384.

52. Carey VJ. Ontology concepts and tools for statistical genomics. J Multivariate Anal. 2004;90(1):213-228.

53. Wall JV, and Jenkins CR. Practical statistics for astronomers. New York, NY: Cambridge University Press; 2003.

54. Benjamini Y, Hochberg Y. Controlling the False Discovery Rate - a Practical and Powerful Approach to Multiple Testing. J Roy Stat Soc B Met. 1995;57(1):289-300.

55. Alterovitz G, Xiang M, Mohan M, Ramoni MF. GO PaD: the Gene Ontology Partition Database. Nucleic Acids Res. 2007;35(Database issue):D322-D327. 